Acta Crystallographica Section E

Structure Reports

Online

ISSN 1600-5368

\section{$\mu$-Acetatato-diacetato $\left\{\boldsymbol{\mu}-6, \mathbf{6}^{\prime}\right.$-dimethoxy- 2,2'-[o-phenylenebis(nitrilomethanylyl- idene)]diphenolato\}gadolinium(III)zinc}

\section{Fan Yang, Guang-Ming Li, Peng Chen, Peng-Fei Yan* and Guang-Feng Hou}

Key Laboratory of Functional Inorganic Materials Chemistry (MOE), School of Chemistry and Materials Science, Heilongjiang University, Harbin, 150080, P. R. China

Correspondence e-mail: yanpf@vip.sina.com

Received 11 July 2011; accepted 18 July 2011

Key indicators: single-crystal X-ray study; $T=293 \mathrm{~K}$; mean $\sigma(\mathrm{C}-\mathrm{C})=0.008 \AA$ : $R$ factor $=0.041 ; w R$ factor $=0.078 ;$ data-to-parameter ratio $=16.9$.

In the heterodinuclear title complex, $\left[\mathrm{GdZn}\left(\mathrm{C}_{22} \mathrm{H}_{18} \mathrm{~N}_{2} \mathrm{O}_{4}\right)\right.$ $\left.\left(\mathrm{CH}_{3} \mathrm{COO}\right)_{3}\right]$, the $\mathrm{Zn}^{\mathrm{II}}$ ion is five-coordinated in a squarepyramidal environment defined by two $\mathrm{O}$ atoms and two $\mathrm{N}$ atoms from the ligand, forming the square plane, and one acetate $\mathrm{O}$ atom serving as the apex, while the $\mathrm{Gd}^{\mathrm{III}}$ ion is ninecoordinated in an approximate mono-capped tetragonalantiprismatic environment defined by four $\mathrm{O}$ atoms from the ligand and five acetate $\mathrm{O}$ atoms.

\section{Related literature}

For the synthesis of the ligand, see: Costes et al. (2000). For similar 3d-4f complexes of similar ligands, see: Bao et al. (2010); Liao et al. (2010); Xu et al. (2011).

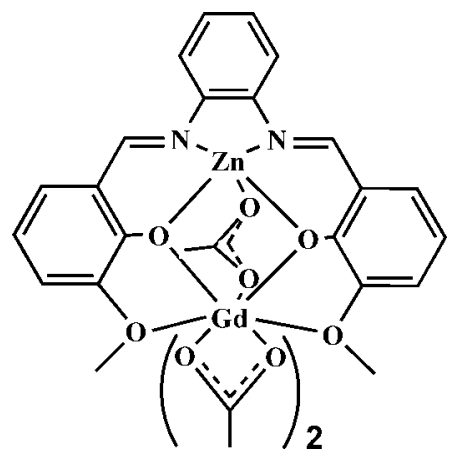

\section{Experimental}

Crystal data

$\left[\mathrm{GdZn}\left(\mathrm{C}_{22} \mathrm{H}_{18} \mathrm{~N}_{2} \mathrm{O}_{4}\right)\left(\mathrm{C}_{2} \mathrm{H}_{3} \mathrm{O}_{2}\right)_{3}\right]$
$M_{r}=774.16$
Monoclinic, $P 2_{1} / c$
$a=14.012(3) \AA$
$b=13.581(3) \AA$
$c=15.426(3) \AA$
$\beta=103.65(3)^{\circ}$

$V=2852.6(10) \AA^{3}$

$Z=4$

Mo $K \alpha$ radiation

$\mu=3.21 \mathrm{~mm}^{-1}$

$T=293 \mathrm{~K}$

$0.15 \times 0.14 \times 0.13 \mathrm{~mm}$

\section{Data collection}

Rigaku R-AXIS RAPID diffractometer

Absorption correction: multi-scan (ABSCOR; Higashi, 1995)

$T_{\min }=0.645, T_{\max }=0.681$

Refinement

$R\left[F^{2}>2 \sigma\left(F^{2}\right)\right]=0.041$

$w R\left(F^{2}\right)=0.078$

$S=1.01$

6488 reflections

384 parameters

$\mathrm{H}$-atom parameters constrained

$\Delta \rho_{\max }=0.59{\mathrm{e} \AA^{-3}}^{-3}$

$\Delta \rho_{\min }=-0.82 \mathrm{e}^{-3}$

26483 measured reflections 6488 independent reflections 4513 reflections with $I>2 \sigma(I)$ $R_{\text {int }}=0.073$

Data collection: RAPID-AUTO (Rigaku, 1998); cell refinement: RAPID-AUTO; data reduction: CrystalClear (Rigaku/MSC, 2002); program(s) used to solve structure: SHELXS97 (Sheldrick, 2008); program(s) used to refine structure: SHELXL97 (Sheldrick, 2008); molecular graphics: SHELXTL (Sheldrick, 2008); software used to prepare material for publication: SHELXL97.

This work was supported financially by the National Natural Science Foundation of China (Nos. 20872030 and 20972043), Heilongjiang Province (Nos. 2009RFXXG201, GC09A402 and 2010 t d03) and Heilongjiang University.

Supplementary data and figures for this paper are available from the IUCr electronic archives (Reference: NG5198).

\section{References}

Bao, Y., Li, G.-M., Yang, F., Yan, P.-F. \& Chen, P. (2010). Acta Cryst. E66, m1379.

Costes, J. P., Dahan, F. \& Dupuis, A. (2000). Inorg. Chem. 39, 5994-6000.

Higashi, T. (1995). ABSCOR. Rigaku Corporation, Tokyo, Japan.

Liao, A., Yang, X. P., Stanley, J. M., Jones, R. A. \& Holiday, B. J. (2010). J. Chem. Crystallogr. 40, 1060-1064.

Rigaku (1998). RAPID-AUTO. Rigaku Corporation, Tokyo, Japan.

Rigaku/MSC (2002). CrystalClear. Rigaku/MSC Inc., The Woodlands, Texas, USA.

Sheldrick, G. M. (2008). Acta Cryst. A64, 112-122.

Xu, L., Li, H.-F., Chen, P. \& Yan, P.-F. (2011). Acta Cryst. E67, m367. 


\section{supporting information}

Acta Cryst. (2011). E67, m1185 [doi:10.1107/S160053681102890X]

\section{$\mu$-Acetato-diacetato $\{\mu$-6,6'-dimethoxy-2,2'-[o-phenylenebis(nitrilomethanylyl- idene)]diphenolato\}gadolinium(III)zinc}

\section{Fan Yang, Guang-Ming Li, Peng Chen, Peng-Fei Yan and Guang-Feng Hou}

\section{S1. Comment}

Lanthanide complexes with spectroscopic and magnetic properties are currently of considerable interest. In continuation of our studies of salen-type lanthanide complexes (Bao et al., 2010, Xu et al., 2011), we present here the synthesis and the crystal structure of the title compound.

In the title compound, the $\mathrm{Zn}$ (II) ion is five-coordinated by two imino nitrogen atoms and two phenolate oxygen atoms from the ligand, and one oxygen from the bridging acetate group to form the pyramid coordination geometry. The $\mathrm{Zn}-\mathrm{N}$ bond distances are in the range of 2.042 (4) $\AA-2.062$ (4) $\AA$, and the $\mathrm{Zn}-\mathrm{O}$ bond distances are in the range of 1.974 (3) $\AA-2.004$ (3) $\AA$, which in accordance with the reported values (Liao et al. 2010). The Gd(III) ion is ligated to five oxygen atoms of three acetate groups, and four oxygen atoms from the ligand. The $\mathrm{Gd}-\mathrm{O}$ bond distances are in the range of 2.365 (3)-2.626 (3) A (Fig.1, Table 1). The Gd-Zn distance is 3.414 (1) $\AA$. The positive charge of the Gd(III) and $\mathrm{Zn}(\mathrm{II})$ ions are balanced by the ligand $L^{2-}$ and three acetate groups ( $L=N, N^{\prime}$-bis(2-oxy-3-methoxybenzylidene)- 1,2-diaminobenzene).

\section{S2. Experimental}

The salen lignad was synthesized following the reference (Costes et al. 2000). To a 1:1 $\mathrm{MeOH} / \mathrm{CH}_{2} \mathrm{Cl}_{2}$ solution (20 ml) of $\mathrm{H}_{2} \mathrm{~L}(0.0748 \mathrm{~g}, 0.2 \mathrm{mmol})$ andGd( $\left(\mathrm{CH}_{3} \mathrm{COO}\right)_{3} \cdot 4 \mathrm{H}_{2} \mathrm{O}(0.0813 \mathrm{~g}, 0.2 \mathrm{mmol})$ was added a MeOH solution (5 ml) of $\mathrm{Zn}\left(\mathrm{CH}_{3} \mathrm{COO}\right)_{2} \cdot 2 \mathrm{H}_{2} \mathrm{O}(0.0438 \mathrm{~g}, 0.2 \mathrm{mmol})$ at the ambient temperature. The color of the solution immediately changed to yellow. After stirring for $8 \mathrm{~h}$, the solution was filtered to remove the suspended particles. yellow single crystals suitable for X-ray diffraction were obtained by slow diffusion of diethylether into the filtrate in four days. $\mathrm{ZnGd}\left(\mathrm{C}_{22} \mathrm{H}_{18} \mathrm{~N}_{2} \mathrm{O}_{4}\right)$ $\left(\mathrm{CH}_{3} \mathrm{COO}\right)_{3}$, Elemental Anal. Calc.: C, 43.44; H, 3.52; N, 3.62 wt\%, Found: C, 43.38; H, 3.44; N, 3.27 wt\%.

\section{S3. Refinement}

The anormal reflection data (-1 14 ) have been omitted during the refinement. $\mathrm{H}$ atoms bound to $\mathrm{C}$ atoms were placed in calculated positions and treated as riding on their parent atoms, with $\mathrm{C}-\mathrm{H}=0.93 \AA$ (aromatic $\mathrm{C}$ ) and $U_{\text {iso }}(\mathrm{H})=$ $1.2 \mathrm{Ueq}(\mathrm{C})$, and with $\mathrm{C}-\mathrm{H}=0.96 \AA$ (methyl $\mathrm{C}$ ) and $U_{\mathrm{iso}}(\mathrm{H})=1.5 \mathrm{Ueq}(\mathrm{C})$. 


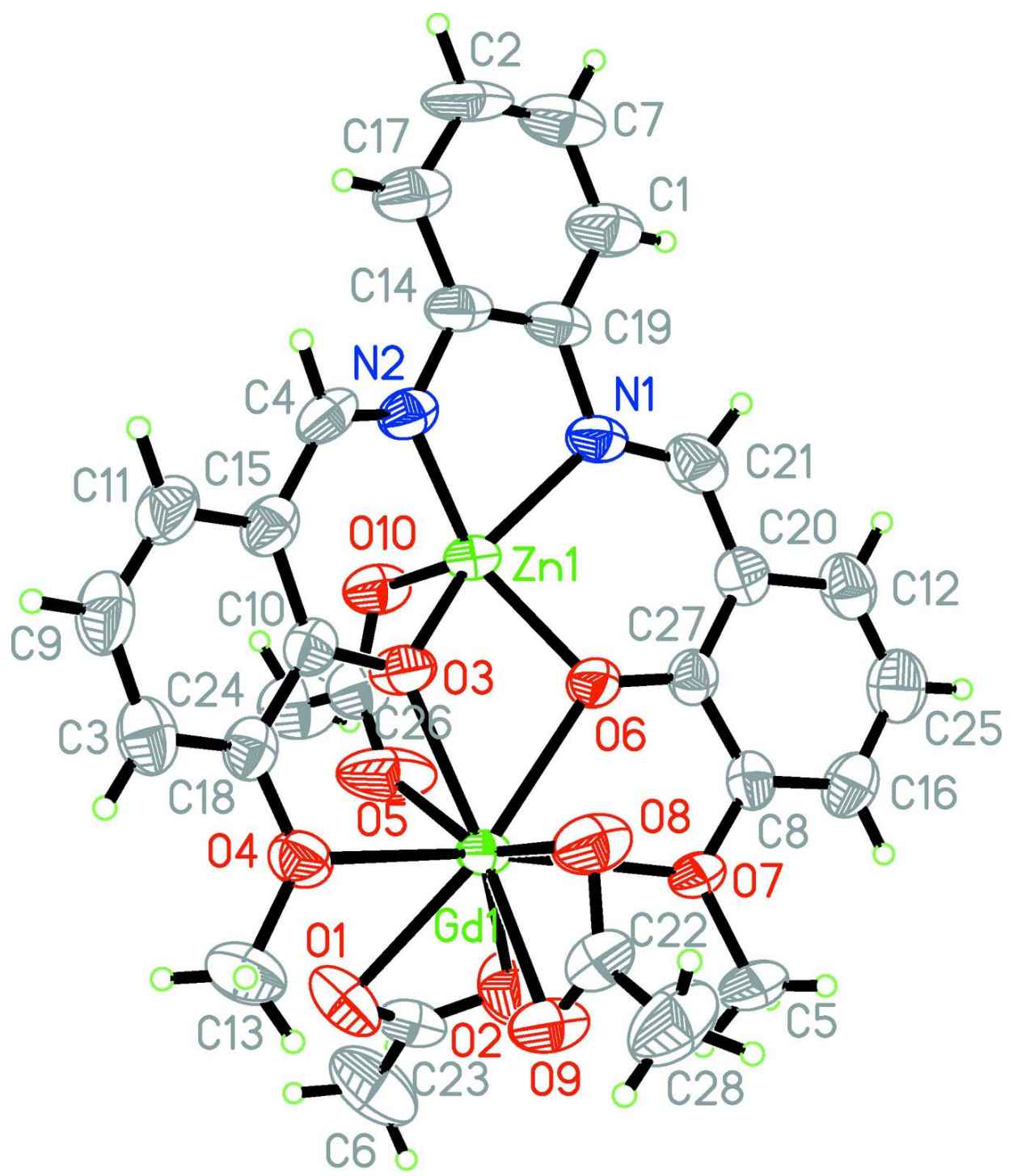

Figure 1

The molecular structure of the title compound, showing $50 \%$ probability displacement ellipsoids.

$\mu$-Acetato-diacetato $\{\mu$-6, 6'-dimethoxy-2,2'-[o-

phenylenebis(nitrilomethanylylidene)]diphenolato\}gadolinium(III)zinc

Crystal data

$\left[\mathrm{GdZn}\left(\mathrm{C}_{22} \mathrm{H}_{18} \mathrm{~N}_{2} \mathrm{O}_{4}\right)\left(\mathrm{C}_{2} \mathrm{H}_{3} \mathrm{O}_{2}\right)_{3}\right]$

$M_{r}=774.16$

Monoclinic, $P 2_{1} / c$

Hall symbol: -P 2ybc

$a=14.012(3) \AA$

$b=13.581(3) \AA$

$c=15.426(3) \AA$

$\beta=103.65(3)^{\circ}$

$V=2852.6(10) \AA^{3}$

$Z=4$

$F(000)=1532$

$D_{\mathrm{x}}=1.803 \mathrm{Mg} \mathrm{m}^{-3}$

Mo $K \alpha$ radiation, $\lambda=0.71073 \AA$

Cell parameters from 16409 reflections

$\theta=3.0-27.5^{\circ}$

$\mu=3.21 \mathrm{~mm}^{-1}$

$T=293 \mathrm{~K}$

Block, yellow

$0.15 \times 0.14 \times 0.13 \mathrm{~mm}$ 


\section{Data collection}

Rigaku R-AXIS RAPID diffractometer

Radiation source: fine-focus sealed tube Graphite monochromator $\omega$ scan

Absorption correction: multi-scan (ABSCOR; Higashi, 1995)

$T_{\min }=0.645, T_{\max }=0.681$

\section{Refinement}

Refinement on $F^{2}$

Least-squares matrix: full

$R\left[F^{2}>2 \sigma\left(F^{2}\right)\right]=0.041$

$w R\left(F^{2}\right)=0.078$

$S=1.01$

6488 reflections

384 parameters

0 restraints

Primary atom site location: structure-invariant direct methods
26483 measured reflections

6488 independent reflections

4513 reflections with $I>2 \sigma(I)$

$R_{\text {int }}=0.073$

$\theta_{\max }=27.5^{\circ}, \theta_{\min }=3.0^{\circ}$

$h=-18 \rightarrow 18$

$k=-17 \rightarrow 16$

$l=-19 \rightarrow 18$

Secondary atom site location: difference Fourier map

Hydrogen site location: inferred from neighbouring sites

$\mathrm{H}$-atom parameters constrained

$w=1 /\left[\sigma^{2}\left(F_{\mathrm{o}}^{2}\right)+(0.0272 P)^{2}+1.1216 P\right]$

where $P=\left(F_{\mathrm{o}}^{2}+2 F_{\mathrm{c}}^{2}\right) / 3$

$(\Delta / \sigma)_{\max }<0.001$

$\Delta \rho_{\max }=0.59$ e $\AA^{-3}$

$\Delta \rho_{\min }=-0.82$ e $\AA^{-3}$

Special details

Geometry. All e.s.d.'s (except the e.s.d. in the dihedral angle between two 1.s. planes) are estimated using the full covariance matrix. The cell e.s.d.'s are taken into account individually in the estimation of e.s.d.'s in distances, angles and torsion angles; correlations between e.s.d.'s in cell parameters are only used when they are defined by crystal symmetry. An approximate (isotropic) treatment of cell e.s.d.'s is used for estimating e.s.d.'s involving 1.s. planes.

Refinement. Refinement of $F^{2}$ against ALL reflections. The weighted $R$-factor $w R$ and goodness of fit $S$ are based on $F^{2}$, conventional $R$-factors $R$ are based on $F$, with $F$ set to zero for negative $F^{2}$. The threshold expression of $F^{2}>\sigma\left(F^{2}\right)$ is used only for calculating $R$-factors(gt) etc. and is not relevant to the choice of reflections for refinement. $R$-factors based on $F^{2}$ are statistically about twice as large as those based on $F$, and $R$-factors based on ALL data will be even larger.

Fractional atomic coordinates and isotropic or equivalent isotropic displacement parameters $\left(\AA^{2}\right)$

\begin{tabular}{lllll}
\hline & $x$ & $y$ & $z$ & $U_{\text {iso }} * / U_{\text {eq }}$ \\
\hline Gd1 & $0.298502(17)$ & $0.880640(16)$ & $0.188340(16)$ & $0.03607(7)$ \\
Zn1 & $0.05908(4)$ & $0.84037(4)$ & $0.19232(4)$ & $0.03815(14)$ \\
O1 & $0.4228(3)$ & $0.9767(3)$ & $0.2964(3)$ & $0.0660(12)$ \\
O2 & $0.4373(3)$ & $0.8188(3)$ & $0.3022(3)$ & $0.0696(12)$ \\
O3 & $0.1371(2)$ & $0.9446(2)$ & $0.1482(2)$ & $0.0457(8)$ \\
O4 & $0.2720(3)$ & $1.0662(2)$ & $0.1405(2)$ & $0.0469(8)$ \\
O5 & $0.2482(3)$ & $0.8884(4)$ & $0.3247(3)$ & $0.0844(14)$ \\
O6 & $0.1715(2)$ & $0.7605(2)$ & $0.1712(2)$ & $0.0448(9)$ \\
O7 & $0.3403(2)$ & $0.6960(2)$ & $0.1719(2)$ & $0.0459(9)$ \\
O8 & $0.2722(3)$ & $0.8700(3)$ & $0.0262(2)$ & $0.0581(10)$ \\
O9 & $0.4224(3)$ & $0.8956(3)$ & $0.1017(3)$ & $0.0594(10)$ \\
O10 & $0.0909(3)$ & $0.8682(2)$ & $0.3216(2)$ & $0.0495(9)$ \\
N1 & $-0.0301(3)$ & $0.7181(3)$ & $0.1660(3)$ & $0.0426(10)$ \\
N2 & $-0.0693(3)$ & $0.9076(3)$ & $0.1312(3)$ & $0.0406(9)$ \\
C1 & $-0.2061(4)$ & $0.6805(5)$ & $0.1540(4)$ & $0.0594(15)$ \\
H1 & -0.1919 & 0.6148 & 0.1686 & $0.071 *$
\end{tabular}




\begin{tabular}{|c|c|c|c|c|}
\hline $\mathrm{C} 2$ & $-0.3228(4)$ & $0.8097(6)$ & $0.1166(4)$ & $0.0722(18)$ \\
\hline $\mathrm{H} 2 \mathrm{~A}$ & -0.3877 & 0.8308 & 0.1047 & $0.087^{*}$ \\
\hline $\mathrm{C} 3$ & $0.1527(5)$ & $1.1987(3)$ & $0.0881(3)$ & $0.0528(14)$ \\
\hline $\mathrm{H} 3 \mathrm{~A}$ & 0.2012 & 1.2443 & 0.0847 & $0.063^{*}$ \\
\hline $\mathrm{C} 4$ & -0.0760 & $0.9959(3)$ & 0.0987 (3) & $0.0441(12)$ \\
\hline $\mathrm{H} 4 \mathrm{~A}$ & -0.1388 & 1.0190 & 0.0729 & $0.053^{*}$ \\
\hline $\mathrm{C} 5$ & $0.4366(4)$ & $0.6643(4)$ & $0.1674(5)$ & $0.0705(18)$ \\
\hline $\mathrm{H} 5 \mathrm{~A}$ & 0.4329 & 0.6302 & 0.1122 & $0.106^{*}$ \\
\hline $\mathrm{H} 5 \mathrm{~B}$ & 0.4626 & 0.6208 & 0.2164 & $0.106^{*}$ \\
\hline $\mathrm{H} 5 \mathrm{C}$ & 0.4788 & 0.7205 & 0.1707 & $0.106^{*}$ \\
\hline C6 & $0.5288(6)$ & $0.9052(5)$ & $0.4231(4)$ & 0.095 \\
\hline H6A & 0.5924 & 0.8835 & 0.4180 & $0.142^{*}$ \\
\hline H6B & 0.5060 & 0.8634 & 0.4642 & $0.142 *$ \\
\hline $\mathrm{H} 6 \mathrm{C}$ & 0.5331 & 0.9718 & 0.4445 & $0.142 *$ \\
\hline $\mathrm{C} 7$ & $-0.3015(5)$ & $0.7124(5)$ & 0.1369 (4) & $0.0728(18)$ \\
\hline $\mathrm{H} 7 \mathrm{~A}$ & -0.3517 & 0.6683 & 0.1390 & $0.087^{*}$ \\
\hline $\mathrm{C} 8$ & $0.2713(4)$ & $0.6248(3)$ & $0.1765(3)$ & 0.0399 (11) \\
\hline C9 & $0.0535(5)$ & $1.2258(4)$ & $0.0651(4)$ & $0.0602(16)$ \\
\hline H9A & 0.0361 & 1.2898 & 0.0466 & $0.072 *$ \\
\hline $\mathrm{C} 10$ & $0.1059(4)$ & $1.0339(3)$ & 0.1223 & 0.0373 \\
\hline C11 & -0.0183 & $1.1587(4)$ & 0.0697 (4) & 0.0550 \\
\hline H11A & -0.0838 & 1.1777 & 0.0532 & $0.066^{*}$ \\
\hline $\mathrm{C} 12$ & $0.1196(5)$ & $0.4954(3)$ & $0.1731(4)$ & $0.0541(14)$ \\
\hline H12A & 0.0680 & 0.4512 & 0.1691 & $0.065^{*}$ \\
\hline C13 & $0.3513(4)$ & $1.1311(4)$ & $0.1360(4)$ & $0.0594(15)$ \\
\hline H13A & 0.3416 & 1.1567 & 0.0765 & $0.089^{*}$ \\
\hline H13B & 0.4122 & 1.0956 & 0.1513 & $0.089^{*}$ \\
\hline $\mathrm{H} 13 \mathrm{C}$ & 0.3531 & 1.1845 & 0.1771 & $0.089^{*}$ \\
\hline C14 & $-0.1514(4)$ & $0.8451(4)$ & 0.1298 & $0.0459(12)$ \\
\hline $\mathrm{C} 15$ & $0.0052(4)$ & $1.0620(3)$ & 0.0987 (3) & $0.0426(12)$ \\
\hline $\mathrm{C} 16$ & $0.2889(4)$ & $0.5246(3)$ & $0.1824(3)$ & $0.0512(13)$ \\
\hline H16A & 0.3522 & 0.5005 & 0.1880 & $0.061^{*}$ \\
\hline $\mathrm{C} 17$ & $-0.2486(4)$ & $0.8776(5)$ & $0.1134(4)$ & 0.0600 \\
\hline H17A & -0.2638 & 0.9435 & 0.1005 & $0.072 *$ \\
\hline C18 & $0.1774(4)$ & $1.1038(3)$ & $0.1156(3)$ & 0.0395 (11) \\
\hline C19 & -0.1301 & $0.7453(4)$ & $0.1496(3)$ & $0.0457(12)$ \\
\hline $\mathrm{C} 20$ & $0.0996(4)$ & $0.5986(3)$ & 0.1719 (3) & $0.0424(12)$ \\
\hline $\mathrm{C} 21$ & $-0.0006(4)$ & $0.6290(4)$ & 0.1625 & $0.0510(13)$ \\
\hline $\mathrm{H} 21 \mathrm{~A}$ & -0.0482 & 0.5798 & 0.1531 & $0.061^{*}$ \\
\hline $\mathrm{C} 22$ & $0.3616(4)$ & $0.8806(4)$ & $0.0284(4)$ & 0.0524 \\
\hline $\mathrm{C} 23$ & $0.4599(4)$ & $0.9003(4)$ & $0.3355(4)$ & $0.0498(13)$ \\
\hline $\mathrm{C} 24$ & $0.2012(5)$ & $0.8960(5)$ & $0.4602(4)$ & $0.0749(18)$ \\
\hline $\mathrm{H} 24 \mathrm{~A}$ & 0.2498 & 0.9466 & 0.4778 & $0.112 *$ \\
\hline H24B & 0.2257 & 0.8352 & 0.4886 & $0.112^{*}$ \\
\hline $\mathrm{H} 24 \mathrm{C}$ & 0.1423 & 0.9144 & 0.4777 & $0.112 *$ \\
\hline $\mathrm{C} 25$ & $0.2112(5)$ & $0.4601(4)$ & $0.1798(4)$ & $0.0632(16)$ \\
\hline $\mathrm{H} 25 \mathrm{~A}$ & 0.2223 & 0.3925 & 0.1827 & $0.076^{*}$ \\
\hline $\mathrm{C} 26$ & $0.1791(4)$ & $0.8833(4)$ & $0.3610(3)$ & $0.0516(13)$ \\
\hline
\end{tabular}


supporting information

\begin{tabular}{lllll}
$\mathrm{C} 27$ & $0.1785(4)$ & $0.6639(3)$ & $0.1738(3)$ & $0.0385(11)$ \\
$\mathrm{C} 28$ & $0.3968(5)$ & $0.8751(5)$ & $-0.0560(4)$ & $0.084(2)$ \\
$\mathrm{H} 28 \mathrm{~A}$ & 0.4423 & 0.9277 & -0.0570 & $0.125^{*}$ \\
$\mathrm{H} 28 \mathrm{~B}$ & 0.3419 & 0.8811 & -0.1064 & $0.125^{*}$ \\
$\mathrm{H} 28 \mathrm{C}$ & 0.4289 & 0.8131 & -0.0586 & $0.125^{*}$ \\
\hline
\end{tabular}

Atomic displacement parameters $\left(\AA^{2}\right)$

\begin{tabular}{|c|c|c|c|c|c|c|}
\hline & $U^{11}$ & $U^{22}$ & $U^{33}$ & $U^{12}$ & $U^{13}$ & $U^{23}$ \\
\hline Gd1 & $0.02645(12)$ & $0.03976(12)$ & $0.04177(14)$ & $-0.00167(10)$ & $0.00757(9)$ & $-0.00027(11)$ \\
\hline Zn1 & $0.0263(3)$ & $0.0438(3)$ & $0.0441(3)$ & $0.0004(2)$ & $0.0079(2)$ & $-0.0002(2)$ \\
\hline $\mathrm{O} 1$ & $0.071(3)$ & $0.054(2)$ & $0.065(3)$ & $-0.028(2)$ & $0.000(2)$ & $0.0017(19)$ \\
\hline $\mathrm{O} 2$ & 0.068 (3) & $0.062(2)$ & $0.064(3)$ & 0.008 (2) & -0.014 (2) & $-0.0066(19)$ \\
\hline $\mathrm{O} 3$ & $0.030(2)$ & $0.0417(18)$ & $0.065(2)$ & $0.0033(14)$ & $0.0124(17)$ & $0.0161(15)$ \\
\hline $\mathrm{O} 4$ & $0.041(2)$ & $0.0432(18)$ & $0.057(2)$ & $-0.0066(15)$ & $0.0117(17)$ & $0.0041(15)$ \\
\hline O5 & 0.045 & $0.166(4)$ & $0.044(2)$ & $-0.021(3)$ & $0.014(2)$ & $-0.010(3)$ \\
\hline O6 & $0.034(2)$ & $0.0337(18)$ & $0.070(3)$ & $-0.0015(14)$ & $0.0186(18)$ & $-0.0013(15)$ \\
\hline $\mathrm{O} 7$ & $0.0256(19)$ & $0.0440(18)$ & $0.066(2)$ & $0.0072(14)$ & $0.0074(16)$ & $-0.0030(15)$ \\
\hline $\mathrm{O} 8$ & $0.041(2)$ & $0.090(3)$ & $0.044(2)$ & $0.004(2)$ & $0.0114(17)$ & $-0.0026(18)$ \\
\hline O9 & $0.036(2)$ & $0.087(3)$ & $0.057(3)$ & $-0.0017(19)$ & $0.0137(19)$ & $0.001(2)$ \\
\hline $\mathrm{O} 10$ & $0.036(2)$ & $0.070(2)$ & $0.043(2)$ & $0.0000(17)$ & $0.0112(16)$ & $0.0014(16)$ \\
\hline N1 & $0.031(2)$ & $0.054(2)$ & $0.044(3)$ & $-0.0075(18)$ & $0.0116(19)$ & $-0.0008(18)$ \\
\hline $\mathrm{N} 2$ & $0.033(2)$ & $0.051(2)$ & $0.037(2)$ & $0.0069(17)$ & $0.0061(18)$ & $-0.0012(17)$ \\
\hline $\mathrm{C} 1$ & $0.041(4)$ & $0.088(4)$ & $0.051(4)$ & $-0.013(3)$ & $0.016(3)$ & $0.000(3)$ \\
\hline $\mathrm{C} 2$ & $0.022(3)$ & $0.134(6)$ & $0.061(4)$ & $-0.009(3)$ & $0.010(3)$ & $-0.007(4)$ \\
\hline $\mathrm{C} 3$ & $0.070(4)$ & $0.039(3)$ & $0.047(3)$ & $0.000(3)$ & $0.008(3)$ & $0.002(2)$ \\
\hline $\mathrm{C} 4$ & $0.034(3)$ & $0.062(3)$ & $0.036(3)$ & $0.017(2)$ & $0.008(2)$ & $-0.005(2)$ \\
\hline $\mathrm{C} 5$ & $0.028(3)$ & 0.069 (4) & $0.112(6)$ & $0.011(3)$ & $0.014(3)$ & $-0.010(3)$ \\
\hline C6 & $0.096(6)$ & $0.110(5)$ & $0.064(5)$ & $-0.045(5)$ & $-0.008(4)$ & $-0.005(4)$ \\
\hline $\mathrm{C} 7$ & $0.040(4)$ & $0.110(5)$ & $0.068(5)$ & $-0.022(3)$ & $0.012(3)$ & $0.000(4)$ \\
\hline $\mathrm{C} 8$ & $0.046(3)$ & $0.036(2)$ & $0.036(3)$ & $0.001(2)$ & $0.007(2)$ & $-0.0035(19)$ \\
\hline $\mathrm{C} 9$ & $0.078(5)$ & $0.038(3)$ & $0.060(4)$ & $0.014(3)$ & 0.008 & $0.001(2)$ \\
\hline C10 & $0.040(3)$ & $0.038(2)$ & $0.032(3)$ & $0.006(2)$ & $0.007(2)$ & $-0.0005(18)$ \\
\hline C11 & $0.050(4)$ & $0.050(3)$ & $0.058(4)$ & $0.017(3)$ & $0.000(3)$ & $-0.002(2)$ \\
\hline $\mathrm{C} 12$ & 0.067 (4) & $0.041(3)$ & 0.057 (4) & $-0.011(3)$ & $0.020(3)$ & $0.001(2)$ \\
\hline $\mathrm{C} 13$ & $0.051(4)$ & $0.063(3)$ & $0.063(4)$ & $-0.017(3)$ & 0.010 & $0.012(3)$ \\
\hline C14 & $0.028(3)$ & $0.069(3)$ & $0.039(3)$ & $-0.001(2)$ & $0.005(2)$ & -0.005 (2) \\
\hline $\mathrm{C} 15$ & $0.042(3)$ & 0.048 (3) & $0.036(3)$ & $0.011(2)$ & $0.005(2)$ & $-0.002(2)$ \\
\hline $\mathrm{C} 16$ & $0.058(4)$ & $0.046(3)$ & $0.050(3)$ & 0.015 & $0.014(3)$ & $0.006(2)$ \\
\hline $\mathrm{C} 17$ & $0.038(3)$ & $0.090(4)$ & $0.050(3)$ & 0.007 (3) & $0.007(3)$ & $-0.002(3)$ \\
\hline $\mathrm{C} 18$ & 0.045 & $0.040(3)$ & $0.031(3)$ & $0.004(2)$ & $0.006(2)$ & $-0.0037(18)$ \\
\hline C19 & 0.029 & $0.066(3)$ & $0.041(3)$ & $-0.010(2)$ & $0.007(2)$ & $-0.004(2)$ \\
\hline $\mathrm{C} 20$ & $0.050(3)$ & $0.041(3)$ & 0.038 & $-0.005(2)$ & $0.016(2)$ & $0.0008(19)$ \\
\hline $\mathrm{C} 21$ & $0.054(4)$ & $0.051(3)$ & $0.051(3)$ & $-0.017(3)$ & 0.018 & $0.000(2)$ \\
\hline $\mathrm{C} 22$ & $0.046(3)$ & 0.059 (3) & $0.056(4)$ & 0.009 (3) & 0.020 & $0.002(3)$ \\
\hline $\mathrm{C} 23$ & $0.036(3)$ & $0.066(4)$ & 0.047 (3) & $-0.015(3)$ & $0.008(2)$ & $0.002(3)$ \\
\hline $\mathrm{C} 24$ & $0.067(5)$ & $0.114(5)$ & $0.043(4)$ & $-0.003(4)$ & $0.011(3)$ & $-0.002(3)$ \\
\hline $\mathrm{C} 25$ & $0.079(5)$ & $0.041(3)$ & $0.071(4)$ & $0.004(3)$ & $0.019(4)$ & $0.006(3)$ \\
\hline
\end{tabular}




\begin{tabular}{lllllll}
$\mathrm{C} 26$ & $0.049(4)$ & $0.066(3)$ & $0.038(3)$ & $0.001(3)$ & $0.006(3)$ & $-0.001(2)$ \\
$\mathrm{C} 27$ & $0.044(3)$ & $0.035(2)$ & $0.038(3)$ & $-0.001(2)$ & $0.013(2)$ & $0.0001(19)$ \\
$\mathrm{C} 28$ & $0.078(5)$ & $0.120(5)$ & $0.061(4)$ & $0.023(4)$ & $0.032(4)$ & $-0.006(4)$ \\
\hline
\end{tabular}

Geometric parameters $\left(A,{ }^{o}\right)$

\begin{tabular}{|c|c|c|c|}
\hline $\mathrm{Gd} 1-\mathrm{O} 3$ & $2.365(3)$ & $\mathrm{C} 4-\mathrm{C} 15$ & $1.449(7)$ \\
\hline $\mathrm{Gd} 1-\mathrm{O} 5$ & $2.373(4)$ & $\mathrm{C} 4-\mathrm{H} 4 \mathrm{~A}$ & 0.9300 \\
\hline $\mathrm{Gd} 1-\mathrm{O} 6$ & $2.382(3)$ & $\mathrm{C} 5-\mathrm{H} 5 \mathrm{~A}$ & 0.9600 \\
\hline $\mathrm{Gd} 1-\mathrm{O} 9$ & $2.436(4)$ & $\mathrm{C} 5-\mathrm{H} 5 \mathrm{~B}$ & 0.9600 \\
\hline $\mathrm{Gd} 1-\mathrm{O} 2$ & $2.441(4)$ & $\mathrm{C} 5-\mathrm{H} 5 \mathrm{C}$ & 0.9600 \\
\hline $\mathrm{Gd} 1-\mathrm{O} 8$ & $2.445(4)$ & $\mathrm{C} 6-\mathrm{C} 23$ & $1.465(8)$ \\
\hline $\mathrm{Gd} 1-\mathrm{O} 1$ & $2.479(4)$ & C6-H6A & 0.9600 \\
\hline $\mathrm{Gd} 1-\mathrm{O} 7$ & $2.601(3)$ & C6-H6B & 0.9600 \\
\hline $\mathrm{Gd} 1-\mathrm{O} 4$ & $2.627(3)$ & C6- $\mathrm{H} 6 \mathrm{C}$ & 0.9600 \\
\hline $\mathrm{Gd} 1-\mathrm{C} 22$ & $2.810(6)$ & C7-H7A & 0.9300 \\
\hline $\mathrm{Gd} 1-\mathrm{C} 23$ & $2.814(5)$ & $\mathrm{C} 8-\mathrm{C} 16$ & $1.382(6)$ \\
\hline $\mathrm{Gd} 1-\mathrm{Zn} 1$ & $3.4139(9)$ & $\mathrm{C} 8-\mathrm{C} 27$ & $1.395(6)$ \\
\hline $\mathrm{Zn} 1-\mathrm{O} 10$ & $1.975(3)$ & $\mathrm{C} 9-\mathrm{C} 11$ & $1.371(8)$ \\
\hline $\mathrm{Zn} 1-\mathrm{O} 6$ & $2.002(3)$ & C9-H9A & 0.9300 \\
\hline $\mathrm{Zn} 1-\mathrm{O} 3$ & $2.003(3)$ & $\mathrm{C} 10-\mathrm{C} 18$ & $1.402(6)$ \\
\hline $\mathrm{Zn} 1-\mathrm{N} 2$ & $2.039(4)$ & $\mathrm{C} 10-\mathrm{C} 15$ & $1.425(6)$ \\
\hline $\mathrm{Zn} 1-\mathrm{N} 1$ & $2.060(4)$ & $\mathrm{C} 11-\mathrm{C} 15$ & $1.401(6)$ \\
\hline $\mathrm{O} 1-\mathrm{C} 23$ & $1.250(6)$ & $\mathrm{C} 11-\mathrm{H} 11 \mathrm{~A}$ & 0.9300 \\
\hline $\mathrm{O} 2-\mathrm{C} 23$ & $1.230(6)$ & $\mathrm{C} 12-\mathrm{C} 25$ & $1.351(8)$ \\
\hline $\mathrm{O} 3-\mathrm{C} 10$ & $1.319(5)$ & $\mathrm{C} 12-\mathrm{C} 20$ & $1.429(6)$ \\
\hline $\mathrm{O} 4-\mathrm{C} 18$ & $1.387(6)$ & $\mathrm{C} 12-\mathrm{H} 12 \mathrm{~A}$ & 0.9300 \\
\hline $\mathrm{O} 4-\mathrm{C} 13$ & $1.434(6)$ & $\mathrm{C} 13-\mathrm{H} 13 \mathrm{~A}$ & 0.9600 \\
\hline $\mathrm{O} 5-\mathrm{C} 26$ & $1.230(7)$ & $\mathrm{C} 13-\mathrm{H} 13 \mathrm{~B}$ & 0.9600 \\
\hline $\mathrm{O} 6-\mathrm{C} 27$ & $1.315(5)$ & $\mathrm{C} 13-\mathrm{H} 13 \mathrm{C}$ & 0.9600 \\
\hline $\mathrm{O} 7-\mathrm{C} 8$ & $1.382(5)$ & $\mathrm{C} 14-\mathrm{C} 17$ & $1.397(7)$ \\
\hline $\mathrm{O} 7-\mathrm{C} 5$ & $1.433(6)$ & $\mathrm{C} 14-\mathrm{C} 19$ & $1.406(7)$ \\
\hline $\mathrm{O} 8-\mathrm{C} 22$ & $1.253(6)$ & $\mathrm{C} 16-\mathrm{C} 25$ & $1.390(8)$ \\
\hline $\mathrm{O} 9-\mathrm{C} 22$ & $1.261(6)$ & $\mathrm{C} 16-\mathrm{H} 16 \mathrm{~A}$ & 0.9300 \\
\hline $\mathrm{O} 10-\mathrm{C} 26$ & $1.259(6)$ & C17-H17A & 0.9300 \\
\hline $\mathrm{N} 1-\mathrm{C} 21$ & $1.284(6)$ & $\mathrm{C} 20-\mathrm{C} 27$ & $1.413(6)$ \\
\hline $\mathrm{N} 1-\mathrm{C} 19$ & $1.413(6)$ & $\mathrm{C} 20-\mathrm{C} 21$ & $1.438(7)$ \\
\hline $\mathrm{N} 2-\mathrm{C} 4$ & $1.295(6)$ & $\mathrm{C} 21-\mathrm{H} 21 \mathrm{~A}$ & 0.9300 \\
\hline $\mathrm{N} 2-\mathrm{C} 14$ & $1.427(6)$ & $\mathrm{C} 22-\mathrm{C} 28$ & $1.499(8)$ \\
\hline $\mathrm{C} 1-\mathrm{C} 7$ & $1.370(8)$ & $\mathrm{C} 24-\mathrm{C} 26$ & $1.498(7)$ \\
\hline $\mathrm{C} 1-\mathrm{C} 19$ & $1.396(7)$ & $\mathrm{C} 24-\mathrm{H} 24 \mathrm{~A}$ & 0.9600 \\
\hline $\mathrm{C} 1-\mathrm{H} 1 \mathrm{~A}$ & 0.9300 & $\mathrm{C} 24-\mathrm{H} 24 \mathrm{~B}$ & 0.9600 \\
\hline $\mathrm{C} 2-\mathrm{C} 7$ & $1.375(8)$ & $\mathrm{C} 24-\mathrm{H} 24 \mathrm{C}$ & 0.9600 \\
\hline $\mathrm{C} 2-\mathrm{C} 17$ & $1.398(8)$ & $\mathrm{C} 25-\mathrm{H} 25 \mathrm{~A}$ & 0.9300 \\
\hline $\mathrm{C} 2-\mathrm{H} 2 \mathrm{~A}$ & 0.9300 & $\mathrm{C} 28-\mathrm{H} 28 \mathrm{~A}$ & 0.9600 \\
\hline $\mathrm{C} 3-\mathrm{C} 18$ & $1.376(6)$ & $\mathrm{C} 28-\mathrm{H} 28 \mathrm{~B}$ & 0.9600 \\
\hline $\mathrm{C} 3-\mathrm{C} 9$ & $1.400(8)$ & $\mathrm{C} 28-\mathrm{H} 28 \mathrm{C}$ & 0.9600 \\
\hline $\mathrm{C} 3-\mathrm{H} 3 \mathrm{~A}$ & 0.9300 & & \\
\hline
\end{tabular}




\begin{tabular}{|c|c|c|c|}
\hline $\mathrm{O} 3-\mathrm{Gd} 1-\mathrm{O} 5$ & $75.50(14)$ & $\mathrm{C} 7-\mathrm{C} 1-\mathrm{C} 19$ & $120.8(6)$ \\
\hline $\mathrm{O} 3-\mathrm{Gd} 1-\mathrm{O} 6$ & $65.12(10)$ & $\mathrm{C} 7-\mathrm{C} 1-\mathrm{H} 1 \mathrm{~A}$ & 119.6 \\
\hline $\mathrm{O} 5-\mathrm{Gd} 1-\mathrm{O} 6$ & 76.04 (13) & $\mathrm{C} 19-\mathrm{C} 1-\mathrm{H} 1 \mathrm{~A}$ & 119.6 \\
\hline $\mathrm{O} 3-\mathrm{Gd} 1-\mathrm{O} 9$ & $125.94(12)$ & $\mathrm{C} 7-\mathrm{C} 2-\mathrm{C} 17$ & $121.3(6)$ \\
\hline $\mathrm{O} 5-\mathrm{Gd} 1-\mathrm{O} 9$ & $151.85(14)$ & $\mathrm{C} 7-\mathrm{C} 2-\mathrm{H} 2 \mathrm{~A}$ & 119.4 \\
\hline $\mathrm{O} 6-\mathrm{Gd} 1-\mathrm{O} 9$ & $127.31(12)$ & $\mathrm{C} 17-\mathrm{C} 2-\mathrm{H} 2 \mathrm{~A}$ & 119.4 \\
\hline $\mathrm{O} 3-\mathrm{Gd} 1-\mathrm{O} 2$ & $149.88(14)$ & $\mathrm{C} 18-\mathrm{C} 3-\mathrm{C} 9$ & $119.1(5)$ \\
\hline $\mathrm{O} 5-\mathrm{Gd} 1-\mathrm{O} 2$ & $74.38(16)$ & $\mathrm{C} 18-\mathrm{C} 3-\mathrm{H} 3 \mathrm{~A}$ & 120.4 \\
\hline $\mathrm{O} 6-\mathrm{Gd} 1-\mathrm{O} 2$ & $106.97(12)$ & $\mathrm{C} 9-\mathrm{C} 3-\mathrm{H} 3 \mathrm{~A}$ & 120.4 \\
\hline $\mathrm{O} 9-\mathrm{Gd} 1-\mathrm{O} 2$ & $82.74(14)$ & $\mathrm{N} 2-\mathrm{C} 4-\mathrm{C} 15$ & $126.0(4)$ \\
\hline $\mathrm{O} 3-\mathrm{Gd} 1-\mathrm{O} 8$ & $81.38(13)$ & $\mathrm{N} 2-\mathrm{C} 4-\mathrm{H} 4 \mathrm{~A}$ & 117.0 \\
\hline $\mathrm{O} 5-\mathrm{Gd} 1-\mathrm{O} 8$ & $154.78(14)$ & $\mathrm{C} 15-\mathrm{C} 4-\mathrm{H} 4 \mathrm{~A}$ & 117.0 \\
\hline $\mathrm{O} 6-\mathrm{Gd} 1-\mathrm{O} 8$ & $85.25(12)$ & $\mathrm{O} 7-\mathrm{C} 5-\mathrm{H} 5 \mathrm{~A}$ & 109.5 \\
\hline $\mathrm{O} 9-\mathrm{Gd} 1-\mathrm{O} 8$ & $53.03(13)$ & $\mathrm{O} 7-\mathrm{C} 5-\mathrm{H} 5 \mathrm{~B}$ & 109.5 \\
\hline $\mathrm{O} 2-\mathrm{Gd} 1-\mathrm{O} 8$ & $128.15(14)$ & $\mathrm{H} 5 \mathrm{~A}-\mathrm{C} 5-\mathrm{H} 5 \mathrm{~B}$ & 109.5 \\
\hline $\mathrm{O} 3-\mathrm{Gd} 1-\mathrm{O} 1$ & $117.37(13)$ & $\mathrm{O} 7-\mathrm{C} 5-\mathrm{H} 5 \mathrm{C}$ & 109.5 \\
\hline $\mathrm{O} 5-\mathrm{Gd} 1-\mathrm{O} 1$ & $71.90(14)$ & $\mathrm{H} 5 \mathrm{~A}-\mathrm{C} 5-\mathrm{H} 5 \mathrm{C}$ & 109.5 \\
\hline $\mathrm{O} 6-\mathrm{Gd} 1-\mathrm{O} 1$ & $145.40(12)$ & $\mathrm{H} 5 \mathrm{~B}-\mathrm{C} 5-\mathrm{H} 5 \mathrm{C}$ & 109.5 \\
\hline $\mathrm{O} 9-\mathrm{Gd} 1-\mathrm{O} 1$ & $81.16(13)$ & $\mathrm{C} 23-\mathrm{C} 6-\mathrm{H} 6 \mathrm{~A}$ & 109.5 \\
\hline $\mathrm{O} 2-\mathrm{Gd} 1-\mathrm{O} 1$ & $51.91(13)$ & $\mathrm{C} 23-\mathrm{C} 6-\mathrm{H} 6 \mathrm{~B}$ & 109.5 \\
\hline $\mathrm{O} 8-\mathrm{Gd} 1-\mathrm{O} 1$ & $129.16(13)$ & $\mathrm{H} 6 \mathrm{~A}-\mathrm{C} 6-\mathrm{H} 6 \mathrm{~B}$ & 109.5 \\
\hline $\mathrm{O} 3-\mathrm{Gd} 1-\mathrm{O} 7$ & $123.52(10)$ & $\mathrm{C} 23-\mathrm{C} 6-\mathrm{H} 6 \mathrm{C}$ & 109.5 \\
\hline $\mathrm{O} 5-\mathrm{Gd} 1-\mathrm{O} 7$ & $104.50(14)$ & $\mathrm{H} 6 \mathrm{~A}-\mathrm{C} 6-\mathrm{H} 6 \mathrm{C}$ & 109.5 \\
\hline $\mathrm{O} 6-\mathrm{Gd} 1-\mathrm{O} 7$ & $60.56(10)$ & $\mathrm{H} 6 \mathrm{~B}-\mathrm{C} 6-\mathrm{H} 6 \mathrm{C}$ & 109.5 \\
\hline $\mathrm{O} 9-\mathrm{Gd} 1-\mathrm{O} 7$ & 79.67 (12) & $\mathrm{C} 1-\mathrm{C} 7-\mathrm{C} 2$ & $119.8(6)$ \\
\hline $\mathrm{O} 2-\mathrm{Gd} 1-\mathrm{O} 7$ & $65.11(11)$ & $\mathrm{C} 1-\mathrm{C} 7-\mathrm{H} 7 \mathrm{~A}$ & 120.1 \\
\hline $\mathrm{O} 8-\mathrm{Gd} 1-\mathrm{O} 7$ & $79.98(11)$ & $\mathrm{C} 2-\mathrm{C} 7-\mathrm{H} 7 \mathrm{~A}$ & 120.1 \\
\hline $\mathrm{O} 1-\mathrm{Gd} 1-\mathrm{O} 7$ & $115.73(12)$ & $\mathrm{O} 7-\mathrm{C} 8-\mathrm{C} 16$ & $125.2(5)$ \\
\hline $\mathrm{O} 3-\mathrm{Gd} 1-\mathrm{O} 4$ & $60.72(10)$ & $\mathrm{O} 7-\mathrm{C} 8-\mathrm{C} 27$ & $113.0(4)$ \\
\hline $\mathrm{O} 5-\mathrm{Gd} 1-\mathrm{O} 4$ & $98.87(14)$ & $\mathrm{C} 16-\mathrm{C} 8-\mathrm{C} 27$ & $121.8(5)$ \\
\hline $\mathrm{O} 6-\mathrm{Gd} 1-\mathrm{O} 4$ & $124.96(11)$ & $\mathrm{C} 11-\mathrm{C} 9-\mathrm{C} 3$ & $120.6(5)$ \\
\hline $\mathrm{O} 9-\mathrm{Gd} 1-\mathrm{O} 4$ & $80.67(12)$ & $\mathrm{C} 11-\mathrm{C} 9-\mathrm{H} 9 \mathrm{~A}$ & 119.7 \\
\hline $\mathrm{O} 2-\mathrm{Gd} 1-\mathrm{O} 4$ & $124.60(12)$ & $\mathrm{C} 3-\mathrm{C} 9-\mathrm{H} 9 \mathrm{~A}$ & 119.7 \\
\hline $\mathrm{O} 8-\mathrm{Gd} 1-\mathrm{O} 4$ & $78.02(11)$ & $\mathrm{O} 3-\mathrm{C} 10-\mathrm{C} 18$ & $117.0(4)$ \\
\hline $\mathrm{O} 1-\mathrm{Gd} 1-\mathrm{O} 4$ & $73.41(12)$ & $\mathrm{O} 3-\mathrm{C} 10-\mathrm{C} 15$ & $124.1(4)$ \\
\hline $\mathrm{O} 7-\mathrm{Gd} 1-\mathrm{O} 4$ & $156.55(11)$ & $\mathrm{C} 18-\mathrm{C} 10-\mathrm{C} 15$ & $118.8(4)$ \\
\hline $\mathrm{O} 3-\mathrm{Gd} 1-\mathrm{C} 22$ & $104.47(14)$ & $\mathrm{C} 9-\mathrm{C} 11-\mathrm{C} 15$ & $121.3(5)$ \\
\hline $\mathrm{O} 5-\mathrm{Gd} 1-\mathrm{C} 22$ & $177.28(16)$ & $\mathrm{C} 9-\mathrm{C} 11-\mathrm{H} 11 \mathrm{~A}$ & 119.3 \\
\hline $\mathrm{O} 6-\mathrm{Gd} 1-\mathrm{C} 22$ & $106.46(15)$ & $\mathrm{C} 15-\mathrm{C} 11-\mathrm{H} 11 \mathrm{~A}$ & 119.3 \\
\hline $\mathrm{O} 9-\mathrm{Gd} 1-\mathrm{C} 22$ & $26.62(14)$ & $\mathrm{C} 25-\mathrm{C} 12-\mathrm{C} 20$ & $121.9(5)$ \\
\hline $\mathrm{O} 2-\mathrm{Gd} 1-\mathrm{C} 22$ & $105.62(16)$ & $\mathrm{C} 25-\mathrm{C} 12-\mathrm{H} 12 \mathrm{~A}$ & 119.0 \\
\hline $\mathrm{O} 8-\mathrm{Gd} 1-\mathrm{C} 22$ & $26.43(14)$ & $\mathrm{C} 20-\mathrm{C} 12-\mathrm{H} 12 \mathrm{~A}$ & 119.0 \\
\hline $\mathrm{O} 1-\mathrm{Gd} 1-\mathrm{C} 22$ & $105.89(16)$ & $\mathrm{O} 4-\mathrm{C} 13-\mathrm{H} 13 \mathrm{~A}$ & 109.5 \\
\hline $\mathrm{O} 7-\mathrm{Gd} 1-\mathrm{C} 22$ & $77.82(13)$ & $\mathrm{O} 4-\mathrm{C} 13-\mathrm{H} 13 \mathrm{~B}$ & 109.5 \\
\hline $\mathrm{O} 4-\mathrm{Gd} 1-\mathrm{C} 22$ & $78.86(13)$ & $\mathrm{H} 13 \mathrm{~A}-\mathrm{C} 13-\mathrm{H} 13 \mathrm{~B}$ & 109.5 \\
\hline $\mathrm{O} 3-\mathrm{Gd} 1-\mathrm{C} 23$ & $135.50(14)$ & $\mathrm{O} 4-\mathrm{C} 13-\mathrm{H} 13 \mathrm{C}$ & 109.5 \\
\hline $\mathrm{O} 5-\mathrm{Gd} 1-\mathrm{C} 23$ & $68.36(15)$ & $\mathrm{H} 13 \mathrm{~A}-\mathrm{C} 13-\mathrm{H} 13 \mathrm{C}$ & 109.5 \\
\hline
\end{tabular}




\begin{tabular}{|c|c|c|c|}
\hline $\mathrm{O} 6-\mathrm{Gd} 1-\mathrm{C} 23$ & $126.30(13)$ & $\mathrm{H} 13 \mathrm{~B}-\mathrm{C} 13-\mathrm{H} 13 \mathrm{C}$ & 109.5 \\
\hline $\mathrm{O} 9-\mathrm{Gd} 1-\mathrm{C} 23$ & $83.90(14)$ & $\mathrm{C} 17-\mathrm{C} 14-\mathrm{C} 19$ & $119.5(5)$ \\
\hline $\mathrm{O} 2-\mathrm{Gd} 1-\mathrm{C} 23$ & $25.85(13)$ & $\mathrm{C} 17-\mathrm{C} 14-\mathrm{N} 2$ & $124.3(5)$ \\
\hline $\mathrm{O} 8-\mathrm{Gd} 1-\mathrm{C} 23$ & $136.84(14)$ & $\mathrm{C} 19-\mathrm{C} 14-\mathrm{N} 2$ & $116.2(4)$ \\
\hline $\mathrm{O} 1-\mathrm{Gd} 1-\mathrm{C} 23$ & $26.35(12)$ & $\mathrm{C} 11-\mathrm{C} 15-\mathrm{C} 10$ & $118.4(5)$ \\
\hline $\mathrm{O} 7-\mathrm{Gd} 1-\mathrm{C} 23$ & $90.78(13)$ & $\mathrm{C} 11-\mathrm{C} 15-\mathrm{C} 4$ & $117.1(5)$ \\
\hline $\mathrm{O} 4-\mathrm{Gd} 1-\mathrm{C} 23$ & $99.73(13)$ & $\mathrm{C} 10-\mathrm{C} 15-\mathrm{C} 4$ & $124.4(4)$ \\
\hline $\mathrm{C} 22-\mathrm{Gd} 1-\mathrm{C} 23$ & $110.42(16)$ & $\mathrm{C} 8-\mathrm{C} 16-\mathrm{C} 25$ & $119.5(5)$ \\
\hline $\mathrm{O} 3-\mathrm{Gd} 1-\mathrm{Zn} 1$ & $34.95(7)$ & $\mathrm{C} 8-\mathrm{C} 16-\mathrm{H} 16 \mathrm{~A}$ & 120.3 \\
\hline $\mathrm{O} 5-\mathrm{Gd} 1-\mathrm{Zn} 1$ & $59.49(10)$ & $\mathrm{C} 25-\mathrm{C} 16-\mathrm{H} 16 \mathrm{~A}$ & 120.3 \\
\hline $\mathrm{O} 6-\mathrm{Gd} 1-\mathrm{Zn} 1$ & $35.01(7)$ & $\mathrm{C} 14-\mathrm{C} 17-\mathrm{C} 2$ & $119.1(6)$ \\
\hline O9-Gd1-Zn1 & $148.60(9)$ & $\mathrm{C} 14-\mathrm{C} 17-\mathrm{H} 17 \mathrm{~A}$ & 120.5 \\
\hline $\mathrm{O} 2-\mathrm{Gd} 1-\mathrm{Zn} 1$ & $123.47(11)$ & $\mathrm{C} 2-\mathrm{C} 17-\mathrm{H} 17 \mathrm{~A}$ & 120.5 \\
\hline $\mathrm{O} 8-\mathrm{Gd} 1-\mathrm{Zn} 1$ & $95.59(10)$ & $\mathrm{C} 3-\mathrm{C} 18-\mathrm{O} 4$ & $125.9(5)$ \\
\hline $\mathrm{O} 1-\mathrm{Gd} 1-\mathrm{Zn} 1$ & $127.58(10)$ & $\mathrm{C} 3-\mathrm{C} 18-\mathrm{C} 10$ & $121.7(5)$ \\
\hline $\mathrm{O} 7-\mathrm{Gd} 1-\mathrm{Zn} 1$ & $95.30(7)$ & $\mathrm{O} 4-\mathrm{C} 18-\mathrm{C} 10$ & $112.5(4)$ \\
\hline $\mathrm{O} 4-\mathrm{Gd} 1-\mathrm{Zn} 1$ & $94.82(8)$ & $\mathrm{C} 1-\mathrm{C} 19-\mathrm{C} 14$ & $119.5(5)$ \\
\hline $\mathrm{C} 22-\mathrm{Gd} 1-\mathrm{Zn} 1$ & $121.98(13)$ & $\mathrm{C} 1-\mathrm{C} 19-\mathrm{N} 1$ & $124.2(5)$ \\
\hline $\mathrm{C} 23-\mathrm{Gd} 1-\mathrm{Zn} 1$ & $127.37(11)$ & $\mathrm{C} 14-\mathrm{C} 19-\mathrm{N} 1$ & $116.2(4)$ \\
\hline $\mathrm{O} 10-\mathrm{Zn} 1-\mathrm{O} 6$ & $105.65(14)$ & $\mathrm{C} 27-\mathrm{C} 20-\mathrm{C} 12$ & $117.8(5)$ \\
\hline $\mathrm{O} 10-\mathrm{Zn} 1-\mathrm{O} 3$ & $101.36(14)$ & $\mathrm{C} 27-\mathrm{C} 20-\mathrm{C} 21$ & $124.3(4)$ \\
\hline $\mathrm{O} 6-\mathrm{Zn} 1-\mathrm{O} 3$ & $79.28(13)$ & $\mathrm{C} 12-\mathrm{C} 20-\mathrm{C} 21$ & $117.8(5)$ \\
\hline $\mathrm{O} 10-\mathrm{Zn} 1-\mathrm{N} 2$ & $110.22(15)$ & $\mathrm{N} 1-\mathrm{C} 21-\mathrm{C} 20$ & $125.6(5)$ \\
\hline $\mathrm{O} 6-\mathrm{Zn} 1-\mathrm{N} 2$ & $144.04(15)$ & $\mathrm{N} 1-\mathrm{C} 21-\mathrm{H} 21 \mathrm{~A}$ & 117.2 \\
\hline $\mathrm{O} 3-\mathrm{Zn} 1-\mathrm{N} 2$ & $91.09(15)$ & $\mathrm{C} 20-\mathrm{C} 21-\mathrm{H} 21 \mathrm{~A}$ & 117.2 \\
\hline $\mathrm{O} 10-\mathrm{Zn} 1-\mathrm{N} 1$ & $109.55(15)$ & $\mathrm{O} 8-\mathrm{C} 22-\mathrm{O} 9$ & $120.1(5)$ \\
\hline $\mathrm{O} 6-\mathrm{Zn} 1-\mathrm{N} 1$ & $89.71(14)$ & $\mathrm{O} 8-\mathrm{C} 22-\mathrm{C} 28$ & $120.2(5)$ \\
\hline $\mathrm{O} 3-\mathrm{Zn} 1-\mathrm{N} 1$ & $148.98(15)$ & $\mathrm{O} 9-\mathrm{C} 22-\mathrm{C} 28$ & $119.7(5)$ \\
\hline $\mathrm{N} 2-\mathrm{Zn} 1-\mathrm{N} 1$ & $80.92(16)$ & $\mathrm{O} 8-\mathrm{C} 22-\mathrm{Gd} 1$ & $60.3(3)$ \\
\hline $\mathrm{O} 10-\mathrm{Zn} 1-\mathrm{Gd} 1$ & $89.96(10)$ & $\mathrm{O} 9-\mathrm{C} 22-\mathrm{Gd} 1$ & $59.9(3)$ \\
\hline $\mathrm{O} 6-\mathrm{Zn} 1-\mathrm{Gd} 1$ & $43.06(9)$ & $\mathrm{C} 28-\mathrm{C} 22-\mathrm{Gd} 1$ & $177.1(4)$ \\
\hline $\mathrm{O} 3-\mathrm{Zn} 1-\mathrm{Gd} 1$ & $42.55(9)$ & $\mathrm{O} 2-\mathrm{C} 23-\mathrm{O} 1$ & $120.5(5)$ \\
\hline $\mathrm{N} 2-\mathrm{Zn} 1-\mathrm{Gd} 1$ & $133.10(12)$ & $\mathrm{O} 2-\mathrm{C} 23-\mathrm{C} 6$ & $118.3(5)$ \\
\hline $\mathrm{N} 1-\mathrm{Zn} 1-\mathrm{Gd} 1$ & $132.72(12)$ & $\mathrm{O} 1-\mathrm{C} 23-\mathrm{C} 6$ & $121.1(5)$ \\
\hline $\mathrm{C} 23-\mathrm{O} 1-\mathrm{Gd} 1$ & $92.0(3)$ & $\mathrm{O} 2-\mathrm{C} 23-\mathrm{Gd} 1$ & $59.9(3)$ \\
\hline $\mathrm{C} 23-\mathrm{O} 2-\mathrm{Gd} 1$ & $94.3(3)$ & $\mathrm{O} 1-\mathrm{C} 23-\mathrm{Gd} 1$ & $61.7(3)$ \\
\hline $\mathrm{C} 10-\mathrm{O} 3-\mathrm{Zn} 1$ & $125.8(3)$ & $\mathrm{C} 6-\mathrm{C} 23-\mathrm{Gd} 1$ & $167.8(5)$ \\
\hline $\mathrm{C} 10-\mathrm{O} 3-\mathrm{Gd} 1$ & $130.2(3)$ & $\mathrm{C} 26-\mathrm{C} 24-\mathrm{H} 24 \mathrm{~A}$ & 109.5 \\
\hline $\mathrm{Zn} 1-\mathrm{O} 3-\mathrm{Gd} 1$ & $102.50(12)$ & $\mathrm{C} 26-\mathrm{C} 24-\mathrm{H} 24 \mathrm{~B}$ & 109.5 \\
\hline $\mathrm{C} 18-\mathrm{O} 4-\mathrm{C} 13$ & $117.4(4)$ & $\mathrm{H} 24 \mathrm{~A}-\mathrm{C} 24-\mathrm{H} 24 \mathrm{~B}$ & 109.5 \\
\hline $\mathrm{C} 18-\mathrm{O} 4-\mathrm{Gd} 1$ & $119.6(3)$ & $\mathrm{C} 26-\mathrm{C} 24-\mathrm{H} 24 \mathrm{C}$ & 109.5 \\
\hline $\mathrm{C} 13-\mathrm{O} 4-\mathrm{Gd} 1$ & $122.9(3)$ & $\mathrm{H} 24 \mathrm{~A}-\mathrm{C} 24-\mathrm{H} 24 \mathrm{C}$ & 109.5 \\
\hline $\mathrm{C} 26-\mathrm{O} 5-\mathrm{Gd} 1$ & $146.3(4)$ & $\mathrm{H} 24 \mathrm{~B}-\mathrm{C} 24-\mathrm{H} 24 \mathrm{C}$ & 109.5 \\
\hline $\mathrm{C} 27-\mathrm{O} 6-\mathrm{Zn} 1$ & $126.2(3)$ & $\mathrm{C} 12-\mathrm{C} 25-\mathrm{C} 16$ & $120.1(5)$ \\
\hline $\mathrm{C} 27-\mathrm{O} 6-\mathrm{Gd} 1$ & $129.1(3)$ & $\mathrm{C} 12-\mathrm{C} 25-\mathrm{H} 25 \mathrm{~A}$ & 119.9 \\
\hline $\mathrm{Zn} 1-\mathrm{O} 6-\mathrm{Gd} 1$ & $101.93(12)$ & $\mathrm{C} 16-\mathrm{C} 25-\mathrm{H} 25 \mathrm{~A}$ & 119.9 \\
\hline $\mathrm{C} 8-\mathrm{O} 7-\mathrm{C} 5$ & $118.1(4)$ & $\mathrm{O} 5-\mathrm{C} 26-\mathrm{O} 10$ & $125.3(5)$ \\
\hline $\mathrm{C} 8-\mathrm{O} 7-\mathrm{Gd} 1$ & $119.5(3)$ & $\mathrm{O} 5-\mathrm{C} 26-\mathrm{C} 24$ & $117.6(5)$ \\
\hline
\end{tabular}


supporting information

\begin{tabular}{llll}
$\mathrm{C} 5-\mathrm{O} 7-\mathrm{Gd} 1$ & $122.1(3)$ & $\mathrm{O} 10-\mathrm{C} 26-\mathrm{C} 24$ & $117.0(5)$ \\
$\mathrm{C} 22-\mathrm{O} 8-\mathrm{Gd} 1$ & $93.3(3)$ & $\mathrm{O} 6-\mathrm{C} 27-\mathrm{C} 8$ & $116.3(4)$ \\
$\mathrm{C} 22-\mathrm{O} 9-\mathrm{Gd} 1$ & $93.5(3)$ & $\mathrm{O} 6-\mathrm{C} 27-\mathrm{C} 20$ & $125.0(4)$ \\
$\mathrm{C} 26-\mathrm{O} 10-\mathrm{Zn} 1$ & $118.7(3)$ & $\mathrm{C} 8-\mathrm{C} 27-\mathrm{C} 20$ & $118.7(4)$ \\
$\mathrm{C} 21-\mathrm{N} 1-\mathrm{C} 19$ & $123.6(4)$ & $\mathrm{C} 22-\mathrm{C} 28-\mathrm{H} 28 \mathrm{~A}$ & 109.5 \\
$\mathrm{C} 21-\mathrm{N} 1-\mathrm{Zn} 1$ & $125.6(4)$ & $\mathrm{C} 22-\mathrm{C} 28-\mathrm{H} 28 \mathrm{~B}$ & 109.5 \\
$\mathrm{C} 19-\mathrm{N} 1-\mathrm{Zn} 1$ & $110.7(3)$ & $\mathrm{H} 28 \mathrm{~A}-\mathrm{C} 28-\mathrm{H} 28 \mathrm{~B}$ & 109.5 \\
$\mathrm{C} 4-\mathrm{N} 2-\mathrm{C} 14$ & $124.1(4)$ & $\mathrm{C} 22-\mathrm{C} 28-\mathrm{H} 28 \mathrm{C}$ & 109.5 \\
$\mathrm{C} 4-\mathrm{N} 2-\mathrm{Zn} 1$ & $124.7(3)$ & $\mathrm{H} 28 \mathrm{~A}-\mathrm{C} 28-\mathrm{H} 28 \mathrm{C}$ & 109.5 \\
$\mathrm{C} 14-\mathrm{N} 2-\mathrm{Zn} 1$ & $111.2(3)$ & $\mathrm{H} 28 \mathrm{~B}-\mathrm{C} 28-\mathrm{H} 28 \mathrm{C}$ & 109.5 \\
\hline
\end{tabular}

